\title{
RENORMALIZATION AND GAUGE INVARIANCE IN QUANTUM ELECTRODYNAMICS
}

\author{
Th. A. J. MARIS, D. DILLENBURG * and Gerhard JACOB* \\ Institulo de Fisica and Faculdade de Filosofia \\ Unilersidade Federal do Rio Grande do Sul. \\ Pôrto Alegre, Brasil
}

Received 8 December 1969

\begin{abstract}
The connection between the field theory and the perturbation expansion of quantum electrodynamics is studied. As a starting point the usual Lagrangian is taken but with the bare electron mass and the renormalization constant $Z_{3}$ set equal to zero. This theory is essentially equivalent to the usual one; however, it does not contain any constant of nature and is dilatational and gauge invariant. both invariances being spontaneously broken. The various limiting procedures implied by the differentiation. the multiplication and the renormalization of the field operators in the Lagrangian are combined in a gauge invariant way to a single limit. Propagator equations are derived which are the usual renormalized ones. except for: (i) a natural cancellation of the quadratic divergence of the vacuum polarization: (ii) the presence of an effective cutoff at $p \approx \epsilon^{-1}$; (iii) the replacement of the renormalization constants $Z_{1}$ and $Z_{2}$ by one gauge dependent function $Z\left(\epsilon^{2}\right)$ : (iv) the limit $\epsilon \rightarrow 0$ which has to be taken. The value $Z(0)$ corresponds to the usual constants $Z_{1}$ and $Z_{2}$. It is expected that in general $Z(0)=0$, but this poses no problem in the present formulation. It is argued that the function $Z\left(\epsilon^{2}\right)$. which is determined by the equations, may render the vacuum polarization finite. One may eliminate the renormalization function from the propagator equations and then perform the limit $\epsilon \rightarrow 0$; this results in the usual perturbation series. However. the renormalization function is essential for an understanding of the high momentum behaviour and of the relation between the field theory and the perturbation expansion.
\end{abstract}

\section{INTRODUCTION}

\subsection{General remarks}

At the present time only one good working quantum field theory is known which might be of a fundamental nature, namely the quantum electrodynamics (QED) of the charged leptons. However, though it is possible to obtain from this theory several accurate predictions which have been well confirmed by experiments [1], the connection between the basic formulation of QED as a field theory and the results of calculations is far from straightforward. It is the purpose of this paper to study this connection more closely. A better understanding of how the field theory of QED operates is

* Senior Research Fellow, Conselho Nacional de Pesquisas. 
of interest on its own right in the study of leptons and could also be helpful for the construction of field theories describing other elementary particles.

It might be worthwhile to explicite the argument for the possible importance of the study of QED for elementary particle physics. In spite of the impressive successes of the more recent descriptions of strongly interacting particles, it is expected that a detailed dynamical approach will eventually be needed. For this purpose the assumption that the asymptotic behaviour of a system of particles far away from their interaction region should be understood from the laws governing their behaviour at small relative distances seems to us more natural than the opposite hypothesis of a pure $S$-matrix theory. The only dynamical theory which is at present available for a description of the behaviour at small relative distances appears to be quantum field theory. One way of studying such a theory is to start from some general assumptions as in the method of axiomatic field theory. It is however not very reassuring that just QED does not fulfill all mathematical axioms usually required in this general quantum field theory. like positive definiteness of the Hilbert space or locality.

It seems therefore important to understand better how nature operates in the only realistic theory of interacting fields which is known, namely in QED. In this theory one has the advantage that there is very little freedom for assumptions, because their consequences can be verified rather immediately. A related advantage of studying such a realistic theory is that one may be able or even compelled to make an unusual conclusion because it is unavoidable*. In model field theories which are based on abstract assumptions, one will have the tendency to put the burden of any unexpected result on the artificial input.

Of course, QED has in the past been the model for practically all quantum field theories which have been studied. We believe however that there is still more to learn from it and this is the motivation for the present investigation. From this point of view, it is encouraging to see that the study of QED has recently again lead to results which are applicable elsewhere. We have in mind the problem of spontaneously broken symmetries (SBS). As was first remarked by Heisenberg and by Nambu, it could be possible that the approximate symmetries observed in nature reflect exact passive symmetries of the basic theory [2,3]. In this way the observed asymmetry could be just a property of the solutions and not of the fundamental equations which they obey. In spite of some initial successes such interpretations have lost the general support, mainly because one did not observe the conserved currents and massless bosons expected from general arguments [4] which were confirmed in several models [5]. A study of a suitable $\mathrm{U}_{2} \otimes \mathrm{U}_{2}$ symmetric formulation of the QED of electrons and muons showed however that in this realistic SBS theory neither the SBS currents are conserved nor Goldstone bosons occur [6-8]. It is in particular inter-

\footnotetext{
* A classic example: The conclusions which lead to modern physics were at the time so unusual that they could almost only have been made in theories (thermodynamics and electrodynamics) which were understood to such a degree that there was hardly any freedom left to draw other conclusions.
} 
There are two other aspects in which our treatment differs from the conventional one. We take the bare mass of the fermion equal to zero. Experience has shown $[21,22]$ that this is probably a necessary condition for the mass renormalization to be finite and the resulting dilatational invariance of the theory is attractive from a general point of view [23]. Furthermore we will take $Z_{3}=0$ from the start, i.e. we do not include a term cor responding to the free Maxwell field in the Lagrangian [24|. In order not to introduce all modifications simultaneously we shall in the next section consider the implications of these last two assumptions for the usual formalism in QED. In the third section the new limiting procedure is discussed. In the subsequent section we derive the modified propagator equations and the relation of our approach to the usual perturbation theory; some additional remarks are presented in the last section. An application of the present ideas to a discussion of the fermion propagator is given in ref. [25]. Throughout the paper, unless otherwise stated, we shall use the notation of ref. $|26|$.

\section{THE USUAL THEORY BUT WITH VANISHING BARE ELECTRON MASS AND $Z_{3}=0$}

We first review briefly the usual treatment of QED [9]. The Lagrangian is given by

$$
\mathcal{L}(x)=\bar{\psi}^{(\mathrm{u})}(x)\left(i \neq-m^{(\mathrm{u})}\right) \psi^{(\mathrm{u})}(x)+\mathcal{L}_{\mathrm{o}}\left(A^{(\mathrm{u})}\right)-e^{(\mathrm{u})} \psi^{(\mathrm{u})}(x) A^{(\mathrm{u})}(x) \psi^{(\mathrm{u})}(x) .
$$

Here and in the following, where necessary, proper symmetrization and subtraction of vacuum expectation values are understood. The superscripts (u) stand for "unrenormalized". The canonical quantization leads to the well-known equal time commutation rules.

In order that the asymptotic fermion field and the transverse photon propagator have the normalizations usual for the free fields and to exhibit the phenomenological coupling constant, renormalized quantities are introduced by

$$
\begin{aligned}
\psi=Z_{2}^{-\frac{1}{2}} \psi^{(\mathrm{u})}, & j^{\nu}=Z_{1} \psi \gamma^{\nu} \psi=Z_{1} Z_{2}^{-1} \psi^{(\mathrm{u})} \gamma^{\nu} \psi^{(\mathrm{u})}, \\
A_{\nu}=Z_{3}^{-\frac{1}{2}} A_{\nu}^{(\mathrm{u})}, & e=Z_{1}^{-1} Z_{2} Z_{3}^{\frac{1}{2}} e^{(\mathrm{u})} .
\end{aligned}
$$

The Lagrangian expressed in these quantities is

$$
\mathcal{L}(x)=Z_{2} \bar{\psi}(x)\left(i \not-m^{(\mathrm{u})}\right) \psi(x)+Z_{3} \mathcal{L}_{\mathrm{o}}(A)-e A_{\nu}(x) j^{\nu}(x)=\mathcal{L}_{\mathrm{o}}(x)+\mathcal{L}^{\prime}(x),
$$




$$
\begin{aligned}
& \mathscr{L}_{\mathrm{o}}(x)=\bar{\psi}(x)(i \not \nexists-m) \psi(x)+\mathcal{L}_{\mathrm{o}}(A), \\
& \mathcal{L}^{\prime}(x)=\left(Z_{2}-1\right) \bar{\psi}(x)(i \not \supset-m) \psi(x)+\left(Z_{3}-1\right) \mathcal{L}_{\mathrm{o}}(A) \\
& +Z_{2} \bar{\psi}(x)\left(m-m(\mathbf{u}) \psi(x)-e A_{\nu}(x) j^{\nu}(x),\right.
\end{aligned}
$$

where $m$ is the experimental mass. The factors $Z$ occur of course also in the equal time commutation rules of the renormalized fields.

Using the decomposition of the Lagrangian as given in eqs. (3) and (4) the interaction representation is set up. In the graphical expansion the effect of all terms in $L^{\prime}(x)$ except $e A_{\nu} j^{\nu}$ can be included in the propagators and vertices multiplying these with factors $Z_{2}^{-1}, Z_{3}^{-1}$ and $Z_{1}$. These factors are absorbed in the definition of the full propagators and vertices and occur therefore explicitly only in the free ones. For instance in ref. [26] it is shown how these remaining factors can be eliminated to give the perturbation expansion in renormalized quantities.

In this section we want first to discuss the mass renormalization, reviewing known results, and then come to the problem of $Z_{3}$.

The mass renormalization turns out to be infinite in any finite order perturbation calculation. More precisely, the selfmass correction $m-m(\mathrm{u})$ caused by the interaction is given by a logarithmically divergent integral times $m$. In practice one absorbs $m^{(\mathrm{u})}$ and its correction in the fermion propagator, adjusting the pole to the experimental mass value, and does not worry anymore about the meaning of the unrenormalized mass. The infinite multiplicative correction to $m$ (u) suggests however that this bare mass has to be taken zero $[27,24,23,28]$ and a self-consistent calculation $[21,22]$ supports this viewpoint. It is very interesting to observe that with $m(\mathrm{u})=0$ no constant of length dimension occurs anymore in the basic theory. A finite non-zero fermion mass can only come about by the spontaneous breaking of the dilatational invariance. This point and its implications for elementary particle physics in general are discussed in refs. $[23,28,29]$. In the following we shall take $m^{(\mathrm{u})}=0$.

With respect to $Z_{3}$, all perturbation calculations which have been performed up to now seem to indicate that this quantity vanishes. We shall take $Z_{3}=0$ from the start; from eq. (3) this means that we omit the term in the Lagrangian corresponding to the free Maxwell field. Such a " $Z_{3}=0$ approach" has been discussed earlier, e.g. in refs. [30, 24].

The Lagrangian now becomes

$$
\mathcal{L}(x)=Z_{2} \bar{\psi}(x) i \not \supset \psi(x)-e Z_{1} \bar{\psi}(x) A(x) \psi(x) .
$$

We keep at present the usual equal time commutation rules for the $\psi$-field and the ones for the $A$-field at different space points. The equation

$$
\frac{\partial A^{\nu}}{\partial x^{\nu}}=0,
$$

is taken as a subsidiary condition. 
From the assumed commutation rules, the subsidiary condition and the field equations follow [31] for all $x$ and $y$

$$
\begin{aligned}
& {\left[\frac{\partial A^{\mu}}{\partial x^{\mu}}, A^{\nu}(y)\right]=i a \frac{\partial}{\partial y_{\nu}}-D(x-y),} \\
& {\left[\frac{\partial A^{\mu}}{\partial x^{\mu}}, \frac{\partial A^{\nu}}{\partial y^{\nu}}\right]=0,} \\
& {\left[\frac{\partial A^{\mu}}{\hat{\imath} x^{\mu}}, \psi(y)\right]=e a D(x-y) \psi(y),} \\
& {\left[\frac{\partial A^{\mu}}{\partial x^{\mu}}, \bar{\psi}(y)\right]=-e a D(x-y) \bar{\psi}(y) .}
\end{aligned}
$$

The constant $a$ determines the gauge and will be discussed later in this section. Here we remark that eqs. (7) are derived ones and that $a$ plays in this derivation the rôle of an integration constant and does not occur in the gauge invariant basic theory.

We adopt the Gupta-Bleuler formalism [9] and to see the connection between the descriptions with different values of $a$ we consider the following formal operator gauge transformations:

$$
\begin{aligned}
\psi^{\prime}(x) & =\mathrm{e}^{-i e(b-1) O^{-}(x)} \psi(x) \mathrm{e}^{-i e(b-1) O^{+}(x)}, \\
A_{\nu}^{\prime}(x) & =A_{\nu}(x)+(b-1)-{ }_{i} x^{\nu}
\end{aligned}
$$

with

$$
O(x)=\left.\frac{\partial}{\partial x^{\nu}}\right|^{-1} A^{\nu}(x)=\lim _{\beta \cdots, 0} \int \bar{D}\left(x-x^{\prime}\right) \frac{\partial A^{\nu}\left(x^{\prime}\right)}{\partial x^{\prime} \nu} \mathrm{e}^{-\beta \mid x_{\mathrm{O}}^{\prime}} \mathrm{d}^{4} x^{\prime} .
$$

The transformed operators are again renormalized [17] as one sees directly by taking the matrix elements between the vacuum and the relevant one particle states. Because $O(x)=0$, the decomposition in positive and negative frequency components $O^{+}$and $O^{-}$is Lorentz invariant. The operation $]^{-1}$ needs a more careful discussion $[17,31]$; we intend to come back to this point at a later time. At present one may alternatively consider $O(x)$ as being defined by eqs. (9).

From the given commutation rules and the formal definition of $O(x)$ one finds 


$$
\begin{aligned}
{\left[O(x), A^{\nu}(y)\right] } & =i a \frac{\partial}{\partial y_{\nu}}-\Gamma_{x}^{-1} D(x-y), \\
{\left[O^{+}(x), O^{-}(y)\right] } & =0, \\
{\left[O^{ \pm}(x), \psi(y)\right] } & =\mp \text { iea }[]_{x}^{-1} D^{ \pm}(x-y) \psi(y), \\
{\left[O^{ \pm}(x), \bar{\psi}(y)\right] } & = \pm \text { iea } a]_{x}^{-1} D^{ \pm}(x-y) \psi(y) .
\end{aligned}
$$

Taking $Z_{1}=Z_{2}$ and ignoring an infinite renormalization factor caused by the multiplication of the local field operators at one point, to which we shall return in sect. 3 , it may be seen that the Lagrangian (5) is invariant under the transformations (8). One may note that this is not the case for the usual Lagrangian because the free Maxwell Lagrangian cannot be chosen to be of the manifestly gauge-invariant form $F^{\mu \nu} F_{\mu \nu}$; as is well known, such a choice gives through the field equations a contradiction with the commutation rules of the charge density with the $\psi$-field.

The photon propagator may be derived from the conventional DysonSchwinger equation

$$
Z_{3} D=D_{\mathrm{o}}-D_{\mathrm{o}} e^{2} \Pi D
$$

with the vacuum polarization

$$
\Pi^{\mu \nu}(k)=-\left(g^{\mu \nu} k^{2}-k^{\mu} k^{\nu}\right) \Pi\left(k^{2}\right) \text {. }
$$

For $Z_{3}=0$ follows from eqs. (10) and (11) that one has to take

$$
D_{\mathrm{o}}^{\mu \nu}(k)=-\left(g^{\mu \nu}-\frac{k^{\mu} k^{\nu}}{k^{2}}\right) k^{-2},
$$

and that

$$
e^{2} \Pi_{\rho}^{\mu}(k) D^{\rho \nu}(k)-\left(g^{\mu \nu}-\frac{k^{\mu} k^{\nu}}{k^{2}}\right)=0,
$$

which represents an equation for the photon propagator.

Eq. (12) also results from the field equation

$$
-\frac{\delta L}{\delta A_{\mu}(x)}=J^{\mu}(x)=\frac{\partial}{\partial x_{\mu}} \square \Lambda(x),
$$

where the Lagrange multiplying operator $\Lambda(x)$ appears as a consequence of the subsidiary condition (6). Because from eq. (13) $J^{\mu}$ has no transverse part, it cannot be the electromagnetic current; we shall come back to this point.

To see how eqs. (12) and (13) are related consider the graphical expansion of 


$$
\left\langle 0 T\left\{\psi(x) J^{\mu}(y) \psi(z)\right\} \mid 0\right\rangle .
$$

One has in momentum space, using eq. (13)

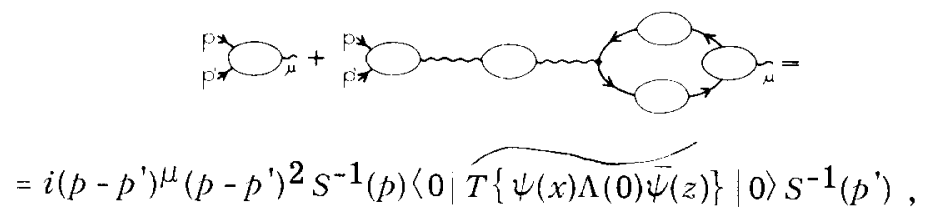

in the usual notation; in the r.h.s. the Fourier transform of the $T$-product should be taken. Eq. (14) can be written

$$
\begin{aligned}
& {\left[g^{\mu \nu}-e^{2} \Pi_{\rho}^{\mu}\left(p-p^{\prime}\right) D^{\rho \nu}\left(p-p^{\prime}\right)\right] \Gamma_{\nu}\left(p, p^{\prime}\right)} \\
& =i\left(p-p^{\prime}\right)^{\mu}\left(p-p^{\prime}\right)^{2} S^{-1}(p)\left\langle 0 \widetilde{T\{\psi \Lambda \bar{\psi}\}|0\rangle S^{-1}\left(p^{\prime}\right) .}\right.
\end{aligned}
$$

Multiplying eq. $\left(14^{\prime}\right)$ with $\left(p-p^{\prime}\right)_{\mu}$ and summing over $\mu$, one obtains a condition on the Lagrange multiplier $\Lambda(x)$. A multiplication with

$$
g^{\sigma \mu}-\frac{\left(p-p^{\prime}\right)^{\sigma}}{\left(p-p^{\prime}\right)^{2}} \frac{\left(p-p^{\prime}\right)^{\mu}}{}
$$

gives, together with eq. (12), an identity. Graphically one may see that eq. (12) and suitable conditions on $\Lambda(x)$, are sufficient for the vacuum expectation of the $T$-product of $J(x)-\lambda \Lambda \partial x_{\mu}$ with any set of field operators to vanish, i.e. for $J_{(x)}^{\mu}-\partial \Lambda / \partial x_{\mu}$ to vanish. One has to take the coordinates of the other operators in a $T$-product with $J^{\mu}(x)$ always different from $x$ in order to be able to interchange the $\epsilon$-limit, which defines $J^{\mu}(x)$ (see sect. 3 ), with the $T$-ordering*. It is however easy to see that a $T$-product, which vanishes for all values of each relative coordinate argument, except if all four components of one of these arguments are zero, vanishes identically.

The solution of eq. (12) is

$$
D^{\mu \nu}(k)=-\left(g^{\mu \nu}-\frac{k^{\mu} k^{\nu}}{k^{2}}\right) e^{-2} \Pi^{-1}\left(k^{2}\right) k^{-2}-a k^{\mu} k^{\nu} k^{-4} .
$$

The term $a k^{\mu} k^{\nu} k^{-4}$ comes about in the following way: The solution of eq. (12) contains an arbitrary additive term $k^{\mu} k_{k}{ }^{-2} D_{l}(k)$ because this component is annihilated by the $g^{\mu \nu} k^{2}-k^{\mu} k^{\nu}$ factor occuring in $I^{\mu \nu}(k)$. The form $k^{\mu} k^{\nu} k^{-4}$ is compelled by the subsidiary condition (6) and the constant $a$ is the same as the one occurring in the commutation rules (7).

Of course, the arbitrariness in the solution for $D^{\mu \nu}(k)$ does at this point not yet necessarily mean that all values of $a$ are in fact allowed. This question depends also on the other Dyson-Schwinger equations and on the physical interpretation. In fact the special value $a=0$ (Landau gauge)

* This is the reason why the lowest order interaction does not vanish. 
is excluded, as this would from eqs. (7) mean that $? v^{\nu}$ vanishes because its vacuum expectation value is zero and it is well known that $? \nu^{\nu} A^{\nu}=0$ does not lead to an acceptable local theory (see eq. (16)). If however one positive value of $a$ is possible (as in the Fermi gauge, where $a=1$ ), then all positive values are allowed. This follows from the gauge invariance of the theory and the fact that under gauge transformations $a$ is multiplied by a positive number. To see this one may investigate the effect of the gauge transformation (8) on the commutation rules (7). Using eqs. (7) and (9) one obtains for the transformed operators again the rules (7) but with a new parameter $a^{\prime}=a b^{*}$. The transformation (8) with $b=0$ evidently transforms any gauge into the Landau gauge, in agreement with ${ }^{2} \nu_{A} 1=0$ following from eq. (8) with $b=0$. Clearly the gauge transformation with $b=0$ has no inverse and therefore does not belong to the invariance group, which is non-compact with a parameter range $0<b<\infty$. This point and the effect of the gauge transformation on the fermion propagator is discussed in ref. [25].

As the gauge transformation (8) leaves the basic theory invariant, but changes the propagators, it cannot be represented in Hilbert space by a unitary transformation which leaves the vacuum invariant. It is therefore a spontaneously broken symmetry (SBS). Solution (15) is a non-normal solution of eq. (12); the normal, non-physical, solution is given by $a=0$, the Landau gauge solution. The possible mathematically non-equivalent irreducible representations, which are typical for a SBS [32], may in this case be labeled by the parameter $\alpha$. The gauge transformation (8) leads from representation $a$ to representation $a b$. Observable quantities are invariant (i.e. independent of the representation), as is required in any SBS theory for the uniqueness of the physical interpretation. We expect that a closer investigation will show that the scalar photons play the rôle of Goldstone bosons, essentially because the symmetry current is conserved in this case.

We have already seen that $J^{\mu}(x)$ of eq. (13) cannot serve anymore as the electric current. In any given irreducible representation $a$ we define the current by

$$
j^{\mu}(x)=A^{\mu}-\left(1-\frac{1}{a}\right) \frac{\lambda}{\partial x_{\mu}} \partial^{\nu} x^{\nu} .
$$

This current is conserved and invariant under the transformation (8). Furthermore $\int j_{0} \mathrm{~d}^{3} x$ has from eqs. (7) the usual commutation rules with the $\psi$ and $A$ fields. The definition (16) shows the unphysical character of the Landau gauge.

The exact formal gauge invariance of the Lagrangian (5) explains the fact that QED shows so many of the properties of a SBS theory with a conserved

* This relation is not trivial and was wrongly given in original manuscript. As pointed out to us by J.A.Swieca. one has to use that. from eq. (9). $x^{-1} D(x-y)$ is not a function of $(x-y)$ and that

$$
\left.\square_{x}^{-1} D^{(} \quad{ }^{(}(x-y)-\square_{y}^{-1} D^{(\quad)}(y-x)=\lim _{m \rightarrow 0}-\frac{\partial}{\partial m^{2}} \Delta_{m}^{(}\right)(x-y)
$$


symmetry current. We mention the gauge dependence of the description, the invariance of observable quantities, the degeneracy of the vacuum and the presence of the long range Coulomb interaction or scalar massless bosons.

If one introduces in the Lagrangian (5) an unrenormalized field $A^{(\mathrm{u})}$ by the definition

$$
A^{\nu(\mathrm{u})}(x)=e A^{\nu}(x),
$$

no constant of nature occurs anymore in the theory. After having solved this form of the theory, the coupling constant $e$ would be found to be the renormalization constant necessary in eq. (17) to renormalize the photon propagator. The coupling constant is therefore, without the transformation (17), determined by the condition that the photon propagator is renormalized, that is from eq. (15)

$$
e^{2} \Pi(0)=1 .
$$

To have a realistic approximation of QED it is at least necessary to introduce also the muon field. This can be achieved by doubling the number of components of the fermion field, introducing an isotopic spin formalism $[33,34]$. By this modification one still has not introduced a constant of nature in the Lagrangian. It is clear that to be realistic the vacuum polarization in eq. (18) certainly should also contain the contributions from the muon field. For a discussion on the possibility of understanding in principle the ratio of the electron and muon masses in such an approach see refs. $[27,23,28,33]$.

The present formulation still contains the weaknesses of the usual formulation of QED. To begin with, one has a quadratic divergence in the photon propagator which has to be subtracted on the basis of a general gauge invariance argument. Of course, after one has started from a gauge invariant Lagrangian, it should not be necessary to invoke gauge invariance again as an extra condition. As we shall see, the reason for this lack of consistency is that the Lagrangian (5) is only formally gauge invariant, but is not actually so, as long as one has not stated how the occuring singular products of field operators should be defined. Furthermore, and much more serious, the electron self-energy and the charge renormalization, i.e. essentially the value of the l.h.s. of eq. (18), turn out to be logarithmically divergent with a possible exception for the electron self-energy in a very special gauge $[21,22]$. We used the word "possible" because it seems to us likely that this special gauge is exactly the forbidden Landau gauge; this problem will be treated elsewhere [25]. It is therefore necessary to improve the definition of the Lagrangian (5), and we shall now turn to this problem.

\section{INTERACTION DEFINITION, DIFFERENTIATION AND RENORMALI- ZATION}

First the problem of the quadratic divergence of the vacuum polarization will be discussed along the lines of refs. $[18,19]$. 
From general arguments it has been shown [35-38] that the electromagnetic current has to contain an explicit dependence on the vector potential. Furthermore it is well known that a product of the type $\bar{\psi}(x) \gamma \mu \psi(x)$ should be defined by a limiting procedure because of the singular properties of the local field operators. The gauge invariant definition of the current $[39,11]$

$$
j^{\mu}(x)=\lim _{\epsilon \rightarrow 0} \frac{1}{4}\left\{\left[\bar{\psi}(x-\epsilon), \gamma^{\mu} \psi(x+\epsilon)\right] \times \exp \left\{-i e \int_{x-\epsilon}^{x+\epsilon} A^{\nu}(s) \mathrm{d} s_{\nu}\right\}+(\epsilon \rightarrow-\epsilon)\right\} \text {, }
$$

has the required dependence on the $A$-field and its use in the field equations appears to include automatically [40] the subtraction terms necessary to cancel the quadratic divergence of the vacuum polarization.

This procedure to get rid of the quadratic divergence is however still not quite straightforward. Namely if the current is taken to be explicitly dependent on $A^{\mu}$, then the interaction term in the Lagrangian density cannot be of the generally assumed form $j_{\mu}(x) A^{\mu}(x)$, as this would result in

$$
j_{\nu}(x)=-\frac{\delta L}{\delta A^{\nu}(x)}=j_{\nu}(x)+A^{\mu}(x) \frac{\partial j_{\mu}(x)}{\partial A^{\nu}},
$$

which contradicts $\partial j_{\mu} / \partial A^{\nu} \neq 0$. Therefore the definition (19) requires a redefinition of the Lagrangian.

A suitable Lagrangian density has been given in refs. [18, 19]; for our case with $m(\mathrm{u})=0, Z_{3}=0$ this definition reduces to

$$
\mathcal{L}(x)=\sum_{\nu \in \rightarrow 0} \lim _{2 \epsilon} \frac{h\left(x, \epsilon \hat{n}^{(\nu)}\right)_{+}^{+}}{2 \epsilon} \text {-vac. exp. val. }
$$

with

$$
h(x, \epsilon \hat{n})=-\bar{\psi}(x+\epsilon \hat{n}) i \gamma \hat{n} \varphi(x, \epsilon \hat{n}) \psi(x-\epsilon \hat{n}),
$$

and $\hat{n}^{(\nu)}$ being a unit vector in the direction of the $v$ th axis. The two sign indices of $h$ indicate here and in the following the part of $h$ which is symmetric under the two commuting operations of charge conjugation (upper index) and hermitian conjugation (lower index), i.e.

$$
h_{+}^{+}=\frac{1}{4}\left(h+h^{\mathrm{c}}+h^{\dagger}+h^{\dagger \mathrm{c}}\right) \text {. }
$$

The operator $\varphi$ is essentially given by

$$
\varphi(x, \epsilon \hat{n})=T_{\epsilon} \exp \left\{-i e \int_{-\epsilon}^{\epsilon} \hat{n} \cdot A\left(x+\epsilon^{\prime} \hat{n}\right) \mathrm{d} \epsilon^{\prime}\right\},
$$

where $T_{\epsilon}$ means time-ordering when $\epsilon$ is a positive number and anti-timeordering when $\epsilon$ is negative. With this convention, which is suggested by gauge invariance, one has $\varphi^{\dagger}(x, \epsilon \hat{n})=\varphi(x,-\epsilon \hat{n})$ and therefore $h \hat{\dagger}(x, \epsilon \hat{n})=$ $-h(x,-\epsilon \hat{n})$. We shall come back to the definition of $\varphi$ after having made some more general remarks. 
The Lagrangian (21) is, neglecting the singular properties of the field operators, identical to the one discussed in the preceding section. From this naive point of view one would write definition (21), using $h^{\dagger}\left(x, \epsilon \hat{n}^{(\nu)}\right)=$ $-h\left(x,-\epsilon \hat{n}^{(\nu)}\right)$ simply as

$$
\begin{aligned}
& L^{\prime}(x)=\frac{1}{4} \sum\left(\begin{array}{c}
i h\left(x, \epsilon n^{(\nu)}\right) \\
\imath \epsilon
\end{array}\right)_{\epsilon=0}^{+} \\
& \left.\quad=\frac{1}{4}\left\{\left[\bar{\psi},\left(i \overrightarrow{\vec{c}} \mu-e A_{\mu}\right) \gamma^{\mu} \psi\right]+\left[\bar{\psi}_{\gamma} \mu_{(-i \vec{c} \mu}-e A_{\mu}\right) \gamma^{\mu}, \psi\right]\right\} .
\end{aligned}
$$

The line integral in the exponential renders the integral formally gauge invariant also for finite $\epsilon$. As is shown in ref. [19] the non-linearity of the exponential introduces extra graphs, containing vertices with more than one photon line, which precisely cancel the quadratic divergence of the vacuum polarization and make it transversal. In this way the usual ad hoc subtraction or redefinition of the current become unnecessary.

Clearly the definition (21) is not manifestly Lorentz invariant because the direction of the coordinate axes plays a preferred rôle. But exactly the same is true in the definition of the differentiation process occurring in the Lagrangian of any quantum field theory. For example a term of the type $\bar{\psi} \psi \boldsymbol{\psi}$ is in our notation defined by

$$
\bar{\psi} x \psi=\lim _{\epsilon \rightarrow 0} \sum \bar{\psi}(x), \nu \frac{\psi\left(x+\epsilon n^{(\nu)}\right)-\psi\left(x-\epsilon n^{(\nu)}\right)}{2 \epsilon} .
$$

The Lorentz invariance of such an expression implies certain continuity properties of the limit (see for example eq. (33)) and is consequently an assumption concerning the solution of the theory, the validity of which should in principle be verified a posteriori. For a classical field such a verification is trivial, but this is not necessarily true for quantum fields with their infinitely many matrix elements and, by definition, singular properties. We shall return to this point in sect. 4.

Now we come to a discussion of the definitions (19) and (23). One may look at the exponential in a slightly different way, writing

$$
\begin{aligned}
\bar{\psi}(x+\epsilon \hat{n}) T_{\epsilon} \exp \left\{-i e \int_{-\epsilon}^{\epsilon} \hat{n} \cdot A\left(x+\epsilon^{\prime} \hat{n}\right) \mathrm{d} \epsilon^{\prime}\right\} \psi(x-\epsilon \hat{n}) \\
=\bar{\psi}(x) \exp (\epsilon \hat{n} \overleftarrow{\partial}) T_{\epsilon} \exp \left\{-i e \int_{\nu \epsilon^{\cdot}}^{\epsilon} \hat{n} \cdot A\left(x+\epsilon^{\prime} \hat{n}\right) \mathrm{d} \epsilon^{\prime}\right\} \exp (-\epsilon \hat{n} \vec{\partial}) \psi(x) .
\end{aligned}
$$

The identity

$\exp (\epsilon \hat{n} \vec{\partial}) T_{\epsilon} \exp \left\{-i e \int_{-\epsilon}^{\epsilon} \hat{n} \cdot A\left(x+\epsilon^{\prime} \hat{n}\right) \mathrm{d} \epsilon^{\prime}\right\} \exp (-\epsilon \hat{n} \vec{\partial})=\exp \{-2 \epsilon(\stackrel{\leftrightarrow}{\partial}+i e A) \cdot \hat{n}\}$, 
with $\overleftrightarrow{\vec{c}}=\frac{1}{2}(\vec{\partial}-\bar{\partial})$ is easily proven by realizing that both sides obey the same first order differential equation in $\epsilon$, with identical boundary conditions at $\epsilon=0$.

Inserting relation (27) in definition (21), one obtains

$$
h(x, \epsilon \hat{n})=-\bar{\psi}(x) i \gamma \hat{n} \exp \{-2 \epsilon(\ddot{c}+i \epsilon A) \cdot \hat{n}\} \psi(x) .
$$

This form shows immediately the gauge invariance of our definition and also suggests that besides the exponential, there will be other non-linear functions of $\epsilon(\overleftrightarrow{\vec{\partial}}+i e A)$ which might be used in the Lagrangian. This corresponds to the possibility of gauge invariantly smearing the fermion fields instead of displacing them. Because of the insensitivity of the propagator equations against the cutoff in momentum space, to be discussed in sect. 4. we believe that it does not matter which precise function of $\epsilon(\vec{\imath}+i c A)$ one uses*. This situation reminds of the similar one in the non-linear phenomenological Lagrangians of hadron physics [41], with the differences that in our case one may calculate in arbitrarily high order and has to perform the limit $\epsilon \rightarrow 0$.

The definition of $T_{\epsilon} \exp \left\{-i e \int_{-\epsilon}^{\epsilon} \hat{n} \cdot A\left(x+\epsilon^{\prime} \hat{n}\right) \mathrm{d} \epsilon^{\prime}\right\}$ has its problems. In the expansion of the exponential occur products of line integrals, which are not defined because the linear smearing of $A$ is not sufficient. For example, one finds for a free field and $\epsilon>0$

$$
\begin{aligned}
& \left\langle 0\left|T \exp \left\{-i e \int_{-\epsilon}^{\epsilon} \hat{n} \cdot A\left(x+\epsilon^{\prime} \hat{n}\right) \mathrm{d} \epsilon^{\prime}\right\}\right| 0\right\rangle \\
& \quad=\exp \left\{-\frac{1}{2} e^{2} \int_{-\epsilon}^{\epsilon} \mathrm{d} \epsilon^{\prime} \int_{-\epsilon}^{\epsilon} \mathrm{d} \epsilon^{\prime \prime} \hat{n} \mu^{\hat{n}} \nu^{\left.i D_{\mathrm{F}}^{\mu \nu}\left(\epsilon^{\prime}-\epsilon^{\prime 2}\right)\right\} .}\right.
\end{aligned}
$$

The exponent is logarithmically divergent, as one sees taking, for instance,

$$
i D_{\mathrm{F}}^{\mu \nu}(x)=(2 \pi)^{-1} g^{\mu \nu} x^{-2}
$$

More generally one has for different $x_{i}$ 's and sufficiently small $\epsilon_{i}>0$

$$
\begin{aligned}
\left\langle 0\left|T \prod_{i} \varphi\left(x_{i}, \epsilon_{i} \hat{n}^{(i)}\right)\right| 0\right\rangle=\exp \{ & -\frac{1}{2} e^{2} \sum_{k} \sum_{l} \int_{-\epsilon_{k}}^{\epsilon_{k}} \mathrm{~d} \epsilon_{k}^{\prime} \int_{-\epsilon_{l}}^{\epsilon_{l}} \mathrm{~d} \epsilon_{l}^{\prime \prime} \\
& \left.\times \hat{n}_{\mu}^{(k)} \hat{n}_{\nu}^{(l)} i D_{\mathrm{F}}^{\mu \nu}\left(x_{k}-x_{l}+\epsilon_{k}^{\prime} \hat{n}^{(k)}-\epsilon_{l}^{\prime \prime} \hat{n}^{(l)}\right)\right\} .
\end{aligned}
$$

The divergences in the exponent come only from the terms $k=l$; this suggests that a good definition of the exponential may be

* B. Liberman has recently shown that this is indeed the case. 


$$
\varphi^{\prime}=\langle 0|\varphi| 0\rangle^{-1} \varphi \text {. }
$$

For the free field case one has now

$$
\left\langle 0\left|\varphi^{\prime}\right| 0\right\rangle=1 \text {, }
$$

and

$$
\begin{aligned}
\left\langle 0\left|T \prod_{i^{\varphi}}{ }^{(i)}\right| 0\right\rangle=\exp \left\{-e^{2} \sum_{k<l} \sum_{-\epsilon_{k}}^{\epsilon_{k}} \int_{-\epsilon l} \mathrm{~d}_{k}^{\prime} \int_{-\epsilon l}^{\epsilon} \mathrm{d} \epsilon_{l}^{\prime \prime}\right. \\
\left.\quad \times \hat{n}_{\mu}^{(k)} \hat{n}_{\nu}^{(l)} i D_{\mathrm{F}}^{\mu \nu}\left(x_{k}-x_{l}+\epsilon_{k}^{\prime} \hat{n}^{(k)}-\epsilon_{l}^{\prime \prime} \hat{n}^{(l)}\right)\right\},
\end{aligned}
$$

which is finite for different $x_{i}$ 's. In the diagrammatic perturbation expansion, the division by the vacuum expectation value $\langle 0|\varphi| 0\rangle$ means that photon lines coming from the same point should not be contracted; closed loops of the photon propagator do not occur.

Although the definition (30) may seem satisfactory, it gives rise to a new problem because the denominator spoils the gauge invariance of the Langrangian (21). As the gauge invariance is one of our leading principles, this would seem to be a serious trouble. It will however soon become clear that the study of the renormalization leads to a natural formulation in which the gauge invariance is restored.

We shall now discuss the renormalization of the fermion field. If one calculates with the help of the usual graphical technique the fermion self energy and the vacuum polarization, one finds in general logarithmically divergent integrals, as was remarked in sect. 2.

The occurrence of these divergences indicates that the limit in our Lagrangian (21) may not exist and following ref. [20] we first generalize the limiting procedure. The limit $(21)$ is of the general form

$$
\lim _{\epsilon \rightarrow 0} \sum_{\nu} \gamma_{\nu} \frac{Q\left(x, \epsilon n^{(\nu)}\right)-Q\left(x,-\epsilon n^{(\nu)}\right)}{2 \epsilon}
$$

or, naively

$$
\gamma_{\nu}\left(\frac{\partial Q(x, \epsilon)}{\partial \epsilon_{\nu}}\right)_{\epsilon=0}
$$

with $Q=h^{+}=h+h^{\mathrm{c}}$. Let us therefore first consider the expression

$$
\lim _{\epsilon \rightarrow 0} \frac{Q\left(x, \epsilon n^{(\nu)}\right)-Q\left(x,-\epsilon n^{(\nu)}\right)}{2 \epsilon}=\left(\frac{\partial Q(x, \epsilon)}{\partial \epsilon \nu}\right)_{\epsilon=0} \text {. }
$$

A priori it is, of course, not certain whether the theory has a solution for which the limit (32) exists and we shall show that relaxing this condition introduces the renormalization in a natural way. First we define $\partial Q / \partial \epsilon_{\nu}$ (omitting from now on the subscript $\epsilon=0$ ) in an invariant way by 
demanding that for any sufficiently small four-vector $\epsilon$ :

$$
Q(x, \epsilon)-Q(x,-\epsilon)=2 \epsilon \nu\left\{\frac{\partial Q}{\partial \epsilon}+R^{\nu}(\epsilon)\right\}
$$

with $\partial Q / \partial \epsilon_{\nu}$ independent of $\epsilon^{2}$ and

$$
\lim _{\epsilon \rightarrow 0} R^{\nu}(\epsilon)=0
$$

Suppose now that the condition (33) for a certain operator function $Q(x, \epsilon)$ cannot be fulfilled. It might in this case still be possible to define a generlalized gradient $g \partial Q / \partial \epsilon_{\nu}$ at $\epsilon=0$ by weakening eq. (33) to

$$
Z\left(\epsilon^{2}\right)\{Q(x, \epsilon)-Q(x,-\epsilon)\}=2 \epsilon_{\nu}\left\{\frac{g \partial Q}{\partial \epsilon \nu}+R^{\nu}(\epsilon)\right\},
$$

where $Z\left(\epsilon^{2}\right)$ is a function of the Lorentz invariant $\epsilon^{2}$ which may be suitably chosen, keeping otherwise the same conditions. It is easily seen that, if there exists any function $Z\left(\epsilon^{2}\right)$ which gives a finite non-zero result for $g \partial Q / \partial \epsilon_{\nu}$ in eq. (34), then this operator is unique up to a multiplicative constant; we exclude very singular situations. The same is true for the leading dependence of $Z\left(\epsilon^{2}\right)$ in the neighbourhood of $\epsilon=0$. This means that the ratios of all matrix elements of $g \partial Q / \partial \epsilon_{\nu}$ are defined for the given operator function $Q(x, \epsilon)$. In $Q E D$, and in other cases we have considered, this turns out to be sufficient to determine the physics, because the arbitrary multiplicative constant can also be looked upon as resulting from a finite field renormalization under which the predictions of a theory are well known to be invariant.

In connection with the limiting procedure (34), the following points may be noted:

(i) The operation $g \partial / \partial \epsilon^{\nu}$ has not the linear character of the differentiation, i.e., in general

$$
\frac{g \partial\left(Q_{1}+Q_{2}\right)}{\partial \epsilon_{\nu}} \neq \frac{g \partial Q_{1}}{\partial \epsilon_{\nu}}+\frac{g \partial Q_{2}}{\partial \epsilon_{\nu}}
$$

because the leading $\epsilon$-dependence for $\epsilon \rightarrow 0$ of the functions $Z\left(\epsilon^{2}\right)$ belonging to $Q_{1}$ and $Q_{2}$ will in general be different. Similarly, under a unitary transformation

$$
\underset{\partial \epsilon_{\nu}}{g \partial U Q U^{-1}} \neq U \frac{g \partial Q}{\partial \epsilon_{\nu}} U^{-1} \cdot \text { constant },
$$

except when the $Z$-function belonging to $g \partial U Q U^{-1} / \partial \epsilon_{\nu}$ has the same leading $\epsilon$-dependence as the one of $g \partial Q / \partial \epsilon_{\nu}$. In agreement with the well known fact that the renormalization constant $Z_{2}\left(=Z_{1}\right)$ changes by an infinite constant under an operator gauge transformation $[10,17]$, one finds [25] that the leading $\epsilon$-dependence of $Z\left(\epsilon^{2}\right)$ varies with the gauge. From the inequality 
(36) follows then that the operator gauge transformation of the Lagrangian can in general not be represented by a unitary transformation.

(ii) In a free field theory $Z\left(\epsilon^{2}\right)$ may evidently be chosen to be a nonzero constant and the limit (34) reduces to the usual one. In QED it turns out that $Z\left(\epsilon^{2}\right)$ is in a gauge $a$ of the form $\left(m^{2} \epsilon^{2}\right)^{a \alpha} / 4 \pi$, which is nearly a constant for not too small an $\epsilon^{2}$. Therefore, in QED the limit (34) is for calculative purposes often nearly equivalent to the usual one. For field theories of strongly interacting particles the difference is expected to be drastic.

(iii) As remarked, we find that in QED $Z\left(\epsilon^{2}\right) \approx\left(m^{2} \epsilon^{2}\right)^{a \alpha / 4 \pi}$; the usual limit (31) is then clearly infinite. Loosely speaking the denominator $\epsilon$ in eq. (31) is just too small; to obtain a finite answer one should have divided by $\epsilon(1-a \alpha 2 \pi)$ instead. From definition (34) follows that now $Q(x, \epsilon)$ is not defined for light-like $\epsilon$. In this case there exist in principle two different generalized limits, namely for $\epsilon^{2} \rightarrow+0$ and $\epsilon^{2} \rightarrow-0$. At present we shall assume that $Z\left(\epsilon^{2}\right)=Z\left(-\epsilon^{2}\right) \geqslant 0$, which is born out by an explicit calculation $[25]$.

With the introduction of the generalized differentiation, the Lagrangian (21) becomes

$$
\mathcal{L}(x)=\frac{g c h_{\nu}^{+}(x, \epsilon)}{\lambda \epsilon \nu}=\lim _{\epsilon \rightarrow 0} \sum_{\nu} Z\left(\epsilon^{2}\right) \frac{h\left(x, \epsilon n^{(\nu)}\right)_{+}^{+}}{2 \epsilon} .
$$

As always the vacuum expectation value is supposed to be subtracted. In the naive $\epsilon$-limit one obtains

$$
\mathcal{L}(x)=\frac{1}{2} Z(0) \psi(x)(i \vec{x}-e A) \psi(x)+\text { charge conj. },
$$

which is the Lagrangian (5) with $Z_{2}=Z_{1}=Z(0)$. By the combination of the three limiting procedures implied in the differentiation, the operator multiplication and the renormalization, the constants $Z_{1}$ and $Z_{2}$ have become one and the same quantity.

The expression (37) is still not gauge invariant, because of the occurrence of the denominator $\left.0\left|T_{\epsilon} \exp \left\{-i e \int A \mathrm{~d} \epsilon^{\prime}\right\}\right| 0\right\rangle$ which introduces an infinite constant by a gauge transformation. We will now give a slightly different definition which does not have this defect and is still identical to the Lagrangian (5) in the naive $\epsilon$-limit.

Consider the expression

$$
l_{\alpha \beta}(x, \epsilon)=\bar{\psi}_{\alpha}(x+\epsilon) \varphi(x, \epsilon) \psi_{\beta}(x-\epsilon)_{+}^{+},
$$

in which $\epsilon$ is now a four-vector and the sign symbols are those defined after eq. (21). The vacuum expectation value

$$
\left\langle 0\left|l_{\alpha \beta}(x, \epsilon)\right| 0\right\rangle=l_{\alpha \beta}(\epsilon),
$$

is independent of $x$ because of the Lorentz invariance of the vacuum. We consider $l_{\alpha \beta}(\epsilon)$ as a $4 \times 4$ matrix in the spinor indices and define $l_{\alpha \beta}^{-1}(\epsilon)$ by

$$
l_{\alpha \beta}^{-1}(\epsilon) l_{\beta \gamma}(\epsilon)=\delta_{\alpha \gamma} \text {. }
$$


One may now define the Lagrangian by

$$
\mathcal{L}(x)=\lim _{\epsilon \rightarrow 0} \epsilon^{-4} r\left(\epsilon^{2}\right)\left\{\sum_{\nu} l_{\alpha \beta}^{-1}\left(\epsilon \hat{n}^{(\nu)}\right) l_{\beta \alpha}\left(x, \epsilon \hat{n}^{(\nu)}\right)-1\right\},
$$

where the 1 at the r.h.s. is the, now explicitly subtracted, vacuum expectation value and $r\left(\epsilon^{2}\right)$ has to be chosen such that the limit exists. The factor $l_{\alpha \beta}^{-1}\left(\epsilon \hat{n}^{(\nu)}\right)$ eliminates again in a diagrammatic expansion all closed photon loops, including their higher order corrections. In the naive limit $\epsilon \rightarrow 0$ the dominant behaviour of $l_{\alpha \beta}^{-1}(\epsilon)$ is, from the definition (39), given by $S_{\beta \alpha}^{-1}(2 \epsilon)$, that is, by an expression of the type $f\left(\epsilon^{2}\right)\left(\epsilon_{\beta \alpha^{\prime}} \epsilon^{4}\right)^{-1}$. In this limit one finds therefore

$$
\begin{aligned}
& \mathscr{L}(x)=r(0) f(0) \lim _{\epsilon \rightarrow 0} \sum\left(\frac{1}{\epsilon \gamma^{\nu}}\right){ }_{\beta \alpha}\left\{l_{\beta \alpha}\left(x, \epsilon \hat{n}^{(\nu)}\right)-\left\langle 0\left|l_{\beta \alpha}\left(x, \epsilon \hat{n}^{(\nu)}\right)\right| 0\right\rangle\right\} \\
& =Z(0) \lim _{\epsilon \rightarrow 0} \sum_{\nu}^{\bar{\psi}\left(x+\epsilon \hat{n}^{(\nu)}\right) \gamma^{\nu} \varphi^{\prime}(x, \epsilon) \psi\left(x-\epsilon \hat{n}^{(\nu)}\right)_{+}^{+}} \\
& =\frac{1}{2} Z(0) \vec{\psi}(x)(i \overleftrightarrow{\partial}-e A) \psi(x)+\text { charge conj. - vac. exp. val. , }
\end{aligned}
$$

as earlier. The function $r\left(\epsilon^{2}\right)$ in definition (40) is gauge-independent and we suspect that one can take it equal to a constant. For the derivation of the Dyson-Schwinger equations this point is of no direct importance as $r\left(\epsilon^{2}\right)$ is absorbed in the renormalization function $Z\left(\epsilon^{2}\right)$. The Lagrangian (40) has still a somewhat symbolic meaning because of the cancellation of the inf $i-$ nite factors contained in $l$ and $l^{-1}$ as a result of the closed photon loops discussed after eq. (30). At present we do not see much harm caused by this feature. Comparing the definitions (40) and (37) one sees that $Z(\epsilon 2)$ has the meaning

$$
Z^{-1}\left(\epsilon^{2}\right)=\frac{\epsilon^{3}\langle 0|\bar{\psi}(x+\epsilon) \varphi(x, \epsilon) \psi(x-\epsilon)| 0\rangle}{\langle 0|\varphi(x, \epsilon)| 0\rangle} r^{-1}\left(\epsilon^{2}\right)
$$

\section{DERIVATION OF THE DYSON-SCHWINGER EQUATIONS}

In the following the propagator equations are derived in a way which is close to the usual derivation. This has the advantage that it becomes clear why our formulation should contain at least all the results of the usual perturbation theory.

With this purpose in mind, we consider the Lagrangian (37) or (40) as the limit for $\epsilon \rightarrow 0$ of a renormalized theory with finite $\epsilon$. We assume that the usual vanishing equal time commutation rules are true for space distances much larger than $\epsilon$. The dynamics itself determines the type of singularities which occur for $\epsilon \rightarrow 0$ in the commutators at equal spacetime points. For finite $\epsilon$ the Dyson-Schwinger equations contain, of course, $\epsilon$ as a parameter and the equations are not Lorentz invariant. In the limit 
$\epsilon \rightarrow 0$ one obtains formally the usual renormalized form of the equations. It turns out. however, that this limit of the equations does not exist because $Z(0)=0$ and expressions of the type $0 \cdot \infty$ arise. This is a source of inconsistencies in the usual formalism.

On the other hand it is conceivable that the solutions $S_{\epsilon}, D_{\epsilon}$ and $\Gamma_{\epsilon}^{\mu}$ of the equations for finite $\epsilon$ have finite limits $S, D$ and $\Gamma$ for $\epsilon \rightarrow 0$. The same will then be true for the $S$-matrix elements. Just because $S_{\epsilon}, D_{\epsilon}$ and $\Gamma_{\epsilon}^{\mu}$ are renormalized, it is really not surprising that they approach finite limits (which are of course still correctly normalized). Elsewhere [25] it is shown for the case of the fermion propagator that this is indeed a very natural situation. It allows a reproduction of the usual perturbation results and leads to a better understanding of the gauge covariance of the propagator and renormalization "constants", which now may vanish (and in fact do so) without any internal inconsistency.

For our present purpose we ignore all problems connected with the existence of the interaction representation. In the usual way we choose a suitable Lorentz invariant zero order Lagrangian, which we take as the basis of an interaction representation and expand the perturbing Lagrangian (which contains the finite constant $\epsilon$ ) in a power series of $e$ (or $A$ ). In the cutoff region $p^{\mu} \approx \epsilon^{-1}$ an uncertainty occurs in this procedure, caused by the fact that we have to take time-ordered products of a non-local perturbation Lagrangian. As we shall see, the Lorentz invariance of the theory demands anyhow that the detailed behaviour of the occurring integrands at the cutoff region be unessential in the limit $\epsilon \rightarrow 0$ and therefore the mentioned uncertainty is of no importance. We shall use convenient expressions for the propagators in the cutoff region. The result of the perturbation expansion can be represented by Feynman diagrams; the Ward-Takahashi identity may as usual be derived up to arbitrary order in the coupling constant and by summing infinitely many graphs the Dyson-Schwinger equations can be written down. From our Lagrangian one finds the conventional diagrammatic expansion [26], but with the following modifications:

(i) Each full propagator and full vertex has an index $\epsilon$.

(ii) Each explicitly occurring $Z_{1}$ or $Z_{2}$ is replaced by $Z\left(\epsilon^{2}\right)$.

(iii) Each $Z_{1}$ is accompanied by the cutoff factor

$$
b^{\mu}\left(p, p^{\prime}, \epsilon\right)=\frac{\sin \left(p-p^{\prime}\right)^{\mu} \epsilon}{\left(p-p^{\prime}\right)^{\mu_{\epsilon}}} \cos \left[\epsilon\left(p+p^{\prime}\right)^{\mu}\right] .
$$

(iv) The non-linearity in $A$ caused by the exponential (23) occurring in the Lagrangian (37) gives rise to vertices with more than one outgoing photon line. Each extra line is accompanied by a factor $\epsilon$, stemming from the line integral. Therefore in the limit $\epsilon \rightarrow 0$ only sufficiently divergent integrals can contribute. It turns out that the only contributions which survive the limiting procedure $\epsilon \rightarrow 0$ are given by the vacuum polarization diagrams of the type 


$$
\begin{aligned}
\underbrace{}_{k, \nu}=-e^{2} \lim _{\epsilon \rightarrow 0} Z\left(\epsilon^{2}\right) \int_{(2 \pi)^{4}} \mathrm{~d}^{4} p\left\{\delta_{\mu \nu} 2 \epsilon\left(\begin{array}{c}
\sin k^{\nu} \epsilon \\
k^{\nu} \epsilon
\end{array}\right)^{2}\right. \\
\left.\times \sin \left(2 p_{\nu} \epsilon\right) \operatorname{Tr}\left\{\gamma^{\nu} S_{\mathrm{F}}(p)\right\}\right\} .
\end{aligned}
$$

This diagram [19] (which of course also occurs in the expansion (14) if the Lagrangian (37) is used) just cancels the usual quadratic divergence of the vacuum polarization.

One obtains the following Dyson-Schwinger equations:

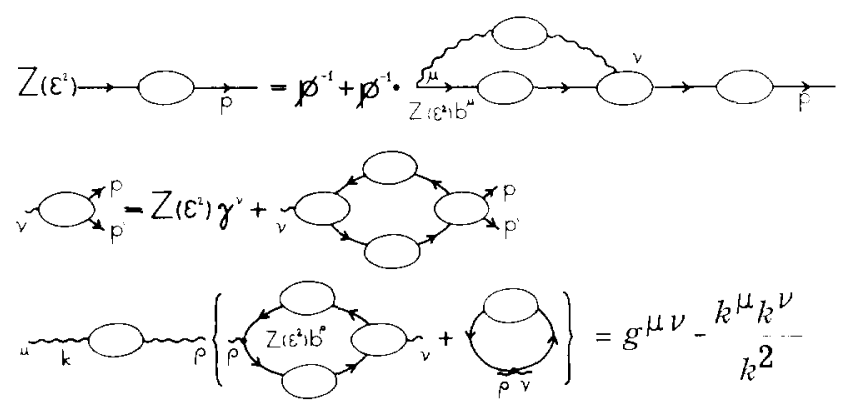

with $b^{\mu}\left(p, p^{\prime}, \epsilon\right)$ and the fermion loop diagram given in eqs. (43) and (44). The other symbols are in the notation of ref. [26], with the understanding that all full propagators, vertices and the kernel have an index $\epsilon$.

In each equation we have left out contributions which obviously vanish for $\epsilon \rightarrow 0$ with at least one power of $\epsilon$ higher than the contributions we have kept.

The equation for the photon propagator is, up to the factor $Z\left(\epsilon^{2}\right)$ and the cutoff, essentially the same as eq. (14') and the remarks following that equation apply.

A remark on the Lorentz non-invariance of the equations caused by the cutoff function $b^{\mu}\left(p, p^{\prime}, \epsilon\right)$ is in order. As was discussed in the preceding section, the Lorentz invariance may be viewed as following from certain continuity conditions put on the solution, which should be verified a posteriori. Let us assume that there are solutions $S_{\epsilon}, D_{\epsilon}$ and $\Gamma_{\epsilon}^{\mu}$ which for $\epsilon \rightarrow 0$ reach certain limits $S, D$ and $\Gamma^{\mu}$. We shall now show that if this is the case, then the details of the cutoff function $b^{\mu}\left(p, p^{\prime}, \epsilon\right)$ do not play a rôle, in the sense that the choice of a different function $b^{\mu}\left(p, p^{\prime}, \epsilon\right)$ will somewhat change the solutions for $S_{\epsilon}, D_{\epsilon}, \Gamma_{\epsilon}^{\mu}$ and $Z\left(\epsilon^{2}\right)$, but not their limits for $\epsilon \rightarrow 0$. Of course the change in the cutoff function has to be made in such a way that the Ward-Takahashi identity is maintained. To see this, one may first observe that the factors $Z\left(\epsilon^{2}\right)$ in eqs. (45) occur so simply that they may be eliminated. One just solves each of the equations for $Z\left(\epsilon^{2}\right)$ or $Z^{-1}\left(\epsilon^{2}\right)$ and, for example, differentiates once towards the free momenta occurring or makes a subtraction. The resulting equations do not contain 
$Z\left(\epsilon^{2}\right)$ anymore. However an integral which diverges for $\epsilon \rightarrow 0$ can only make sense if it is multiplied with $Z\left(\epsilon^{2}\right)$. Therefore if there exists at all a solution, as we are assuming, it should be possible to combine the result ing integrals in such a way that they converge without a cutoff and thus the limit $\epsilon \rightarrow 0$ can be performed separately in $S_{\epsilon}, D_{\epsilon}, \Gamma_{\epsilon}^{\mu}$ and $b^{\mu}(\epsilon)$, giving $S$, $D, \Gamma^{\mu}$ and 1 respectively. Because of the boundary conditions given by the fact that $S, D$ and $\Gamma^{\mu}$ are renormalized, the resulting Lorentz invariant equations will still have unique solutions (of course, up to the multiplicity introduced by the spontaneously broken symmetries as dilatational and gauge invariance). This reasoning shows that the details and in particular the non-Lorentz invariance of $b^{\mu}\left(p, p^{\prime}, \epsilon\right)$ have no influence on the limits of the propagators, when these limits exist at all, although, of course, $S_{\epsilon}$, $D_{\epsilon}, \Gamma_{\epsilon}^{\mu}$ and $Z(\epsilon 2)$ will slightly change with the cutoff function $b$. In certain respects the situation is somewhat similar to the well known one in scattering theory where one determines the density of states by normalizing in a finite box. For this procedure to make any sense at all, the precise boundary conditions must be unimportant, though their existence is essential. The above mentioned procedure in which $Z(\epsilon 2)$ is eliminated may be seen more explicitly in a discussion of the fermion propagator.

\section{CONCLUDING REMARKS}

It appears that the present formulation of the limiting processes in the basic theory, for the moment disregarding our choice of a vanishing bare fermion mass and $Z_{3}=0$, should at least be able to reproduce the experimental predictions of the usual theory. This may be seen in two ways. If one does not take the limit $\epsilon \rightarrow 0$ but chooses $\epsilon$ very small but finite, it seems physically plausible that this gauge invariant non-local unrenormalized version of our approach is quite equivalent to the usual QED, as long as one limits oneself to transitions between states with momenta which are much smaller than $\epsilon^{-1}$. This is because the naive limit $\epsilon \rightarrow 0$ results in the unrenormalized expression $\bar{\psi}(\mathrm{u})(x)(i \not-e A(x) \psi(\mathrm{u})(x)$ of the usual Lagrangian. The finite renormalization of the non-local theory does of course not change this equivalence and the final limit $\epsilon \rightarrow 0$ takes away the restriction put on the considered momenta. Also the propagator equations we derive are for finite $\epsilon$ identical to the usual ones, except for a cutoff and for the replacement of the renormalization constants by functions. All manipulations which are made in the usual theory to eliminate the renormalization constant $Z_{2}\left(=Z_{1}\right)$ may also be performed to eliminate $Z\left(\epsilon^{2}\right)$; the resulting equations are therefore in the limit $\epsilon \rightarrow 0$ identical to the usual renormalized ones.

It is instructive to see why exactly the conventional treatment (see e.g. ref. [26]) is inconsistent. From our point of view one can reproduce this method by taking the limit $\epsilon \rightarrow 0$ in the unrenormalized non-local field theory and only afterwards renormalize the operators. Compared with our treatment one has, for example 


$$
S_{\epsilon}^{(\mathrm{u})}=Z\left(\epsilon^{2}\right) S_{\epsilon}
$$

If however $Z(0)=0$ and $\lim S_{\epsilon}=$ finite, which is found to be the case, one $\epsilon \rightarrow 0$

has

$$
\lim _{\epsilon \rightarrow 0} S_{\epsilon}^{(\mathrm{u})}=0 .
$$

The unrenormalized propagator and therefore also the unrenormalized field operators vanish in the limit $\epsilon \rightarrow 0$. No finite factor will anymore renormalize a quantity once it has vanished; one should have renormalized before going to the limit and that is just what we do.

The present definition of the limiting processes may have some other advantages besides being (hopefully) consistent. The usual limiting procedures in quantum field theories are largely modeled after free field theories and after QED. For the first mentioned case the usual differentiation and multiplication of field operators are identical to the present definition which for this case gives $Z(0)=$ constant $\neq 0$. In QED the renormalization function is nearly also a non-vanishing constant and therefore here the "free" limits are, at least for low momenta, still very good approximations. Because [20]

$$
Z\left(\epsilon^{2}\right) \approx\left(m^{2} \epsilon^{2}\right)^{\alpha / 4 \pi},
$$

$Z\left(\epsilon^{2}\right)$ is of the order one as long as one can take $\epsilon^{2} \gg m^{-2} \exp (-4 \pi \alpha)$. which means for energies up to the order of $m \exp (2 \pi \alpha)$. This is far above any experimental possibility in the foreseeable future. If however a similar situation would be true for strong interactions one finds from eq. (46) replacing $\alpha$ by a strong coupling constant that the usual limiting procedures for interacting hadron fields could well be poor at any energy.

Another basic advantage of using the multiplicative function $Z\left(\epsilon^{2}\right)$ instead of infinite subtractions in a perturbation expansion, appears in the study of the asymptotic behaviour of the propagators. This may be seen in a discussion of the fermion propagator. As remarked earlier [20| also the question of the finiteness of the vacuum polarization seems again to be open. Because $Z(0)=0$, the function $Z\left(\epsilon^{2}\right)$ might be able to cancel the divergence which otherwise occurs in the vacuum polarization if the cutoff is moved to infinite by taking the limit $\epsilon \rightarrow 0$. The expression (46) shows that such a situation might in perturbation theory be reproduced by successive subtractions of logarithmic divergences.

A remarkable feature of the present formulation is that it contains no constant of nature. Whether this implies that the constants occurring in QED, namely the coupling constant and the electron-muon mass ratio, can be calculated in this description depends primarily on the question of whether the isolated theory is, as it stands, really mathematically consistent, or can be made to be so without introducing phenomenological constants. Even if these constants could be calculated, the values found can 
only be satisfactory if the approximation of isolated QED is physically a good one. This seems at present an open question which would have an affirmative answer if the vector dominance model in hadron physics could be taken literally. From the lepton point of view the total effect of the strongly interacting particles would be to create in the photon propagator approximate poles, corresponding to the neutral vector mesons, with residues of the order $\alpha$ as compared to the residue at the photon pole. This would be a small perturbation for the lepton structure, although it would in principle determine the relation of the mass scales of hadrons and leptons $[23,29]$. As long as one does not know more, it seems sound to start with the most simple assumption which appears reasonable, namely to neglect the hadron contributions to the vacuum polarization.

We are thankful for several stimulating discussions we have had with C. G. Bollini, J. J. Giambiagi and J. A. Swieca. The support of the U. S. Army Research Office, the Conselho Nacional de Pesquisas, the Banco Nacional do Desenvolvimento Econômico, the Regional Program of Scientific and Technological Development of the OAS and the Conselho de Pesquisas UFRGS are gratefully acknowledged.

\section{REFERENCES}

[1] W.K.H. Panofsky and S.C.C.Ting, in Proc. 14th int. conf. high-energy physics, Vienna (ed.: J. Prentki and J.Steinberger, CERN, 1968).

[2] W. Heisenberg. Rev. Mod. Phys. 29 (1957) 269.

[3] Y. Nambu and G. Jona-Lasinio, Phys. Rev. 122 (1961) 345.

[4] J.Goldstone, A. Salam and S.Weinberg, Phys. Rev. 127 (1962) 965.

[5] T.W.Kibble, in Proc. 1967 int. conf. particles and fields, Rochester 1967 (ed.: C.R.Hagen, G.Guralnik and V.S. Mathur, Interscience, 1967).

[6] Th.A.J. Maris and Gerhard Jacob, Phys. Rev. Letters 17 (1966) 1300.

[7] K.Johnson, in Proc. 9th Lat. Am. School Phys., Chile 1967 (ed.: I. Saavedra, Benjamin. 1968).

[8] Th. A.J. Maris. Nuovo Cimento Letters 2 (1969) 821.

[9] For a careful discussion and references to the literature, see J.D.Bjorken and S.D. Drell. Relativistic quantum fields (McGraw Hill, 1965);

G. Källen, in Handbuch der Physik, V/1 (ed.: S. Flügge, Springer, 1958) .

[10] K. Johnson and B.Zumino, Phys. Rev. Letters 3 (1959) 351.

[11] J.G.Valatin, Proc. Roy.Soc. A222 (1954) 93 and 228; A225 (1954) 535; A226 (1964) 254

[12] D. G. Boulware, Phys. Rev. 151 (1966) 1024.

[13] R.A. Brandt, Ann. of Phys. 52 (1969) 122.

[14] W.Zimmermann, Com. Math. Phys. 8 (1968) 66; C. de Calan, R.Stora and W.Zimmermann, Nuovo Cimento Letters 1 (1969) 877.

[15] L. D. Landau and I. Khalatnikov, JETP Sov. Phys. 2 (1956) 69.

[16] H.Rollnik, B. Stech and E. Nunnemann, Z. Phys. 159 (1960) 482.

[17] H. Rollnik, Z. Phys. 161 (1961) 370.

[18] Th.A.J.Maris, D. Dillenburg and G.Jacob, Nuovo Cimento 53A (1968) 823.

[19] Th.A.J.Maris, D. Dillenburg, C.E.T.Gonçalves da Silva, G.Jacob and B. Liberman, Nuovo Cimento $55 \mathrm{~A}$ (1968) 590.

[20] Th. A.J.Maris, Nuovo Cimento Letters 1 (1969) 775.

[21] K.Johnson, M. Baker and R. Willey, Phys. Rev. 136 (1964) B1111.

[22] Th.A.J.Maris, V.E.Herscovitz and G.Jacob, Phys. Rev. Letters 12 (1964) 313. 
[23] Th.A.J.Maris, Nuovo Cimento 30 (1963) 378.

[24] R.P. Feynman, in La thérie quantique des champs. Solvay Meeting 1961 (ed.: R. Stoops, Interscience, 1962).

[25] D. Dillenburg and Th.A.J.Maris, Nucl. Phys. B18 (1970) 390.

[26] J. D. Bjorken and S. D. Drell. ref. [9].

[27] M.Baker and S.L.Glashow, Phys. Rev. 128 (1962) 2462.

[28] R. Haag and Th.A.J.Maris. Phys. Rev. 132 (1963) 2325.

[29] Th.A.J.Maris, V.E.Herscovitz and G.Jacob. Nuovo Cimento 38 (1965) 783.

[30] J.-C. Houard and B. Jouvet, Nuovo Cimento 18 (1960) 466.

[31] B. Lautrup, Mat. Fys. Medd. Dan. Vid. Selsk. 35. No. 11.

[32] R. Haag, Nuovo Cimento 25 (1962) 287.

[33] Th.A.J.Maris, V.E. Herscovitz and G.Jacob, Nuovo Cimento 34 (1964) 946.

[34] R. Arnowitt and S. Deser, Phys. Rev, 138 (1965) B712.

[35] J. Schwinger, Phys. Rev. Letters 3 (1959) 296.

[36] K. Johnson, Nucl. Phys. 25 (1961) 431.

[37] L.S. Brown, Phys. Rev. 150 (1966) 1338.

[38] D.G. Boulware and S.Deser, Phys. Rev. 151 (1966) 1278.

[39] P.A. M. Dirac, Proc. Camb. Phil. Soc. 30 (1934) 150.

[40] K. Johnson, in Brandeis Lect. on particles and field theory (ed.: S. Deser and K.W. Ford, Prentice-Hall, 1965).

[41] S.Gasiorowicz and D.A.Geffen, Report DESY 69/13. 
8.B.1 Nuclear Physics B18 (1970) 390-402. North-Holland Publishing Company

\title{
THE FERMION PROPAGATOR OF QUANTUM ELECTRODYNAMICS IN AN ARBITRARY GAUGE
}

\author{
D. DILLENBURG * and Th. A. J. MARIS \\ Instituto de Física and Faculdade de Filosofia \\ Universidade Federal do Rio Grande do Sul \\ Pôrto Alegre, Brasil
}

Receiver 8 December 1969

\begin{abstract}
The fermion propagator equation derived in the preceding paper has been solved using the following approximations: (i) the free photon propagator in an arbitrary gauge is used: (ii) for the vertex a functional of the fermion propagator which approximately obeys the Ward-Takahashi identity is taken: (iii) in the resulting equation a small term is neglected. Closed expressions for the fermion propagator and the renormalization function are obtained in an arbitrary gauge. The gauge dependence found for the fermion propagator is in agreement with the one which follows directly from the gauge transformation of the time ordered product defining the propagator. One recovers the well-known result that $Z_{1}=$ $Z_{2}(=Z(0))$ can only be a finite non-zero number in one special gauge. From a discussion of the operator gauge transformation it seems likely that this finite gauge is non-physical and that in all physical gauges the electron propagator is more singular than the free one and that $Z(0)=0$. In contradistinction to the usual situation this result causes in the present formulation no difficulty and may be essential for the finiteness of the vacuum polarization.
\end{abstract}

\section{INTRODUCTION}

In a recent reformulation $[1,2]$ of the Lagrangian of quantum electrodynamics (QED) the three small distance limiting procedures, implied by the differentiation of the fermion field, by the definition of the interaction and by the renormalization procedure, have been combined in a gauge invariant way. In addition the bare fermion mass and the renormalization constant of the Maxwell field were taken equal to zero. In the following we shall discuss the equation of the fermion propagator resulting from this formulation of the theory.

In sect. 2 we first consider an earlier approximation of the fermion propagator equation in our approach and we show that the exact solution of this equation is finite but has unsatisfactory properties. In sect. 3 an improved ansatz for the vertex is made, resulting in a satisfactory equation which is solved with the free photon propagator in an arbitrary gauge. As

* Senior Research Fellow, Conselho Nacional de Pesquisas. 
expected [3], the solution for the fermion propagator and also the renormalization function $Z\left(\epsilon^{2}\right)$ are dependent on the gauge chosen; in general $Z(0)=0$. A discussion of the results is given in the last section. The gauge dependence of the calculated propagator is shown to be in agreement with the dependence directly found from the operator gauge transformation of the time-ordered product defining the fermion propagator.

\section{DISCUSSION OF AN EARLIER TREATMENT}

Our starting point will be the following form of the Dyson-Schwinger equation for the fermion propagator, derived in refs. [1, 2]:

$$
S^{-1}(p)=Z\left(\epsilon^{2}\right)\left\{p-\frac{i \alpha}{4 \pi^{3}} \int b^{\nu}\left(p, p^{\prime}, \epsilon\right) \gamma^{\nu} S_{\epsilon}\left(p^{\prime}\right) \Gamma_{\epsilon}^{\rho}\left(p^{\prime}, p\right) D \epsilon \nu \rho\left(p^{\prime}-p\right) \mathrm{d}^{4} p^{\prime}\right. \text {. }
$$

The renormalization function $Z\left(\epsilon^{2}\right)$ is determined by the equation itself and

$$
b^{\mu}\left(p, p^{\prime}, \epsilon\right)=\frac{\sin \left(p-p^{\prime}\right)^{\mu_{\epsilon}}}{\left(p-p^{\prime}\right)^{\mu_{\epsilon}}} \cos \left[\epsilon\left(p+p^{\prime}\right)^{\mu}\right] .
$$

Assuming the cutoff independence of the limit of $S_{\epsilon}(p)$ for vanishing $\epsilon$, approximating the vertex by

$$
\Gamma^{\mu}\left(p, p^{\prime}\right)=\frac{1}{2}\left[f\left(-p^{2}\right)+f\left(-p^{\prime 2}\right)\right] \gamma^{\mu}
$$

and taking the free photon propagator in the Fermi gauge, it was shown that

$$
\begin{aligned}
f_{\epsilon}(s) & =Z\left(\epsilon^{2}\right)\left[1+\frac{\alpha}{8 \pi} f_{\epsilon}(s)\left\{s^{-2} \int_{0}^{s f_{\epsilon}^{-1}\left(s^{\prime}\right) s^{\prime 2}} \frac{s^{\prime}+m_{\epsilon}^{2}}{d s^{\prime}}\right.\right. \\
& \left.\left.+\int_{s}^{\epsilon^{-2}} \frac{f_{\epsilon}^{-1}\left(s^{\prime}\right)}{s^{\prime}+m_{\epsilon}^{2}} \mathrm{~d} s^{\prime}\right\}+\frac{\alpha}{8 \pi}\left\{s^{-2} \int_{0}^{s} \frac{s^{\prime 2}}{s^{\prime}+m_{\epsilon}^{2}} \mathrm{~d} s^{\prime}+\int_{s}^{\epsilon^{-2}} \frac{1}{s^{\prime}+m_{\epsilon}^{2}} \mathrm{~d} s^{\prime}\right\}\right],
\end{aligned}
$$

with $s=-p^{2}$ and $S^{-1}(p)=f\left(-p^{2}\right)\left[p-m\left(-p^{2}\right)\right]$. This parametrization of $S$ is somewhat more convenient than the one of ref. [1]. It was remarked that the first terms at the r.h.s. dominate over the two last ones, which were neglected. The resulting equation gave a solution with reasonable properties from a physical point of view.

We shall now show that if one does not neglect the two last terms of eq. (2) one finds a slightly modified solution which is still finite but not anymore quite satisfactory. For this purpose we apply the general method for eliminating $Z\left(\epsilon^{2}\right)$ which was discussed in ref. [2]. Eq. (2) is multiplied by $f_{\epsilon}^{-1}(s)$ and differentiated towards $s$, resulting in 